\title{
Cardiovascular disease and diabetes mellitus in Norway during 1994-2009 CVDNOR - a nationwide research project
}

\author{
Gerhard Sulo $^{1}$, Jannicke Igland ${ }^{1}$, Stein Emil Vollset ${ }^{1,2}$, Ottar Nygård ${ }^{3,4}$, Nina Øyen ${ }^{1,5}$ \\ and Grethe S. Tell ${ }^{1,2}$ \\ 1) Department of Global Public Health and Primary Care, University of Bergen, Bergen, Norway \\ 2) Division of Epidemiology, Norwegian Institute of Public Health, Bergen, Norway \\ 3) Section for Cardiology, Institute of Medicine, University of Bergen, Bergen, Norway \\ 4) Department of Heart Disease, Haukeland University Hospital, Bergen, Norway \\ 5) Center for Medical Genetics and Molecular Medicine, Haukeland University Hospital, Bergen, Norway \\ Correspondence: Gerhard Sulo, Department of Global Public Health and Primary Care, Kalfarveien 31, N-5020 Bergen, Norway \\ E-mail: gerhard.sulo@igs.uib.no Telephone: +4755586100 Telefax: + 4755586130
}

\begin{abstract}
Background: Although having a long tradition in cardiovascular epidemiologic research, nationwide data on cardiovascular disease (CVD) incidence and morbidity has not been available in Norway.

Objectives: The main objectives of The Cardiovascular Disease in Norway (CVDNOR) project are 1) to study trends in CVD incidence, prevalence, survival and mortality and 2) providing CVD endpoints for national and regional health surveys and clinical studies.

Methods: CVDNOR includes information from all hospital stays with a CVD, including congenital heart defects, or diabetes mellitus (DM) primary or secondary diagnosis code during 1994-2009. Information was retrieved from the electronic Patient Administrative Systems (PAS) from all Norwegian somatic hospitals. Data include age, gender, municipality of residence, hospitalization and discharge dates, main and secondary diagnoses and CVD-related procedure codes. All deaths due to CVD or DM are also included, as well as sociodemographic information and linkage to Cohort Norway (CONOR). In sub-projects we have also linked CVDNOR to the Medical Birth Registry of Norway and to the Cancer Registry.

Results: During 1994-2009, 1.3 million patients (4.3 mill hospitalizations) had a CVD diagnosis or procedure code, or DM. Of these, 470895 (35.8\%) died during 1994-2010. In addition, 68523 men and women died from CVD without being hospitalized for CVD or DM during the same period. Among 173243 CONOR participants, 9075 (5\%) died after participating in the health survey and through 2010. A total of 44118 (25.5\%) CONOR participants had a CVD and 7575 (4.4\%) had a DM related hospitalization after participating in CONOR, through 2009. In CVDNOR, 53039 (4\%) had congenital heart defect codes.

Conclusions: CVDNOR will be a valuable source of information supporting future epidemiologic research, with important implications for prevention and treatment strategies.
\end{abstract}

\section{INTRODUCTION}

During the last decades, cardiovascular disease mortality has decreased substantially in Norway $[1,2]$ as in other Scandinavian and western European countries [3-5]. The changes have not been uniform across all sex and age groups [1]. While mortality trends are known in Norway, there is lack of information on trends in incidence and survival. Norway did not have, until 2012, a national register on cardiovascular disease (CVD). This has hampered studies of incidence and survival as well as aetiologic research nationwide.

The Norwegian Patient Register, containing information on all patients hospitalized at Norwegian hospitals since 1990, has the potential to overestimate the true number of hospitalizations and cannot provide information on incidence, due to the lack of personidentifiable information until 2008. Several regional studies have published important information on risk factors [6-8] and occurrence of CVD [9-12]. Never- theless, as shown by the WHO MONICA project, there is a non-negligible difference in occurrence and risk factors for CVD between and even within countries $[13,14]$. Therefore, findings from regional studies may not be generalizable to the general Norwegian population. To fill this gap of knowledge on one of the most important sources of morbidity and mortality in the Norwegian population and elsewhere, the CVDNOR project was planned.

\section{CVDNOR - A PROJECT OFFERING NEW RESEARCH OPPORTUNITIES}

The Cardiovascular Disease in Norway (CVDNOR) project began as a collaborative research project between the University of Bergen and the Norwegian Knowledge Centre for the Health Services. It is a population study with major focus on CVD and DM. The two main objectives are: 
Table 1. Diagnoses and procedures in CVDNOR 1994-2009.

\begin{tabular}{lcc}
\hline Diagnoses included in CVDNOR & ICD9 (1994-1998) & ICD10 (1999-2009) \\
\hline Diseases of the circulatory system & $390-459$ & I00-I99 \\
Oedema, proteinuria and hypertensive complications during pregnancy and childbirth & 642 & O10-O16 \\
Diabetes mellitus during pregnancy & 648.0 & O24 \\
Transient cerebral ischemic attacks and related syndromes & 435 & G 45 \\
Diabetes mellitus & 250 & E10-E14 \\
Non-diabetic hypoglycaemic coma & 251.0 & E15 \\
Sudden, unexpected death & 798.1 & R96 \\
Congenital malformations of the circulatory system & $745-747$ & Q20-Q28 \\
\hline Main diagnostic and treatment procedures & SIF95 & NCMP+ and NCSP \\
\hline Interventions in the heart and great vessels & $3000-3299$ & FA-FY \\
Coronary angiography/left-sided catheterization & $3291,3235^{*}, 3238^{*}$ & FYDB,TFC10, XF911, \\
& & XF912, XF914 \\
Right-sided catheterization & 3290 & TFC00 \\
Electrophysiologic study/intervention of the heart & 3292 & FPA, FPB, FPFE \\
Transthoracic/transesophageal echocardiogram & 3293 & FYDE \\
Percutaneous coronary intervention (PCI) & $3294,3236^{*}, 3239^{*}$ & FNG \\
Coronary artery bypass grafting (CABG) & $3112-3129$ & FNA-FNF \\
Pacemaker/defibrillator implantation procedures & $3200-3209$ & FPE, FPF, FPG \\
Interventions on peripheral blood vessels and lymphatic system & $8800-8899$ & PA-PY \\
\hline
\end{tabular}

* Local codes used by University hospitals Haukeland and Stavanger.

\pm Norwegian classification of medical procedures; $3^{\text {rd }}$ edition, 1995.

† Norwegian classification of medical procedures.

I The NOMESCO classification of surgical procedures; NCMP and NCSP were brought together in 2006 [20].

1. to study trends in CVD incident and recurrent events, short and long term prognosis (survival) and associated factors in the total Norwegian population,

2. to provide CVD and DM endpoints for national and regional health surveys conducted during the last decades in Norway, as well as for clinical studies. CVDNOR will facilitate studies of the impact of several known and potential risk factors (alone and in combination, as well as of changes in risk factors) in CVD occurrence.

\section{Data collection and quality control}

Hospital data

Hospital stays with a CVD (ICD9: 390-459, ICD10: I00-I99, G45), DM (ICD9: 250, ICD10: E10-E14) or congenital malformations of the circulatory system (ICD9: 745-747, ICD10: Q20-Q28) as either primary or secondary diagnosis, as well as all related procedures, were retrieved from the electronic Patient Administrative Systems (PAS) in all Norwegian somatic hospitals from 1994 (the year from which all hospitals adopted an electronic PAS) through 2009. A list of hospitals providing data can be found at www.cvdnor.no. An overview of the main diagnostic and procedural codes eligible for inclusion in CVDNOR is given in Table 1. For hospitalizations containing at least one eligible diagnosis or procedure code, all other diagnosis codes and procedure codes for that stay were also extracted.

A system called FS (Forskning i Sykehus $=$ Research in hospitals) developed by Tomislav Dimoski at the Norwegian Knowledge Centre for the Health Services was used to extract the data retrospectively. The FS-system has been used in studies of other disease entities [15]. It can semi-automatically collect standardized data on patient stays. Patients are identified by use of the Norwegian personal identification number (ID). Transfers between wards, departments or hospitals for the same or different conditions in a patient can therefore be accounted for. A quality control compared FS system-extracted data with patients' hospital records and found that $99 \%$ of the data were correct with regard to date and time of admission and main diagnosis [16].

CVDNOR includes a unique ID-number for each patient and a unique code for each hospitalization. In addition, age at hospitalization, gender, municipality of residence, time and dates of hospitalization and discharge (including transfers between wards and departments within the hospital), hospital, department and ward codes, main and secondary diagnoses (up to 20), medical procedure codes (up to 30 ) performed during the hospital stay, and information about type of hospitalization (acute or elective). Hospitalizations less than 24 hours apart are merged and considered as one.

A more detailed report on CVDNOR data and quality issues will be published shortly at www.cvdnor.no.

Cause of Death Register (www.ssb.no/dodsarsak_en) For all patients identified with a CVD or DM hospitalization and who later died, information about date of death, underlying and contributing cause(s) of death has been retrieved from the Cause of Death Registry. 
In addition, information on individuals who died from CVD or DM but had no previous hospitalizations for CVD or DM was also obtained. Thus, CVDNOR contains data on all CVD or DM related deaths during the study period.

\section{Linkage of hospital data and cause of death data with other sources of information \\ To increase the information obtained and broaden the field of potential research, a series of important linka- ges have been made to other data sources including national registries.}

\section{Statistics Norway, Population Register (www.ssb.no/english)}

Sociodemographic information includes marital status, personal and family income, country of birth, own education, education of spouse, and municipality.

COhort of NORway (CONOR) (www.fhi.no/conor) Since the 1970's, large regional health surveys with a focus on CVD and DM risk factors have been conducted throughout Norway. These surveys were the starting point for the national research collaboration COhort of NORway (CONOR) [17], in which all Norwegian medical faculties (Tromsø, Trondheim, Bergen and Oslo), and the Norwegian Institute of Public Health participated. For the period 1992-2003, the number of participants in CONOR was 173243 , of whom 7460 participated twice.

The linkage of CONOR with CVDNOR provides the opportunity to study the impact of risk factors (e.g. health behaviours, anthropometry, health status) on CVD morbidity and mortality. In addition, changes in risk factors (in those individuals participating twice) and their impact on CVD occurrence may be examined. CONOR data have so far not been used for studies of CVD incidence due to the lack of nationwide morbidity data.

Other health surveys and clinical trials

CVDNOR data will also be used as endpoints in several other health surveys including the Norwegian Counties Studies (www.fhi.no) and the Hordaland Health Studies (http://husk.b.uib.no). In addition, CVDNOR data will be used for extended follow-up of the randomized clinical trials NORVIT [18] and WENBIT [19].

Congenital malformations of the circulatory system, a CVDNOR substudy

The hospital data also include information on congenital malformations of the circulatory system during the same time period. Epidemiologic studies of congenital malformations of the circulatory system have never been undertaken on a national level in Norway due to lack of data. This CVDNOR project will describe the epidemiology of congenital heart defects, such as examining national time trends, risk factors, and mortality of congenital heart defects in Norway. The project also ascertains information on congenital heart defects from the Medical Birth Registry of Norway and from the clinical database on congenital heart defects (Berte) at Oslo University Hospital.

\section{CVDCancer, a CVDNOR substudy}

The purpose of this project is to examine CVD occurrence in cancer survivors. It is known that patients who have been treated for cancer have an excess risk of developing CVD, compared to the population at large. In this project we link CVDNOR to the Norwegian Cancer Registry to study the incidence of CVD in cancer patients compared to the general population. Through linkage to CONOR, risk factors for CVD among CONOR participants with and without cancer will be compared.

Norwegian Prescription Database (www.norpd.no) There are plans to link to the Norwegian Prescription Database (NorPD), although this has not been done yet (as of March 2013). The NorPD contains data about dispensed drugs in Norway, and this linkage will provide the opportunity to examine use of specific medications in relation to incidence and prognosis of CVD and DM. It will also be used to better define disease entities, by exploring the medication use (such as diabetes medication to identify individuals with DM not hospitalized).

\section{Summary of data sources linked to CVDNOR}

Linkages between CVDNOR and other data sources will facilitate longitudinal epidemiologic studies of research questions otherwise not possible to answer. The linked datasets will provide the opportunity for future research projects, and will be a valuable resource for epidemiologic studies.

\section{Data security}

Data from different sources are linked by Statistics Norway. The personal identification number (unique for each Norwegian resident) is replaced by a project specific and unique identification number (ID) before data files are forwarded to the University of Bergen. Data from the different sources are stored as separate datasets on a secure Windows server at the University of Bergen. The unique ID makes it possible to link the different data files. Researchers with given privileges may $\log$ on to the server via a 'Remote Desktop' solution. The Statistical packages SAS, SPSS, STATA and $\mathrm{R}$ are installed on the server in addition to standard software such as Microsoft Office. Data processing is usually done on the server.

Researchers are permitted access only to those data sources that are needed for each specific project.

\section{Ethical considerations and approvals}

The CVDNOR project, including the sub-project on congenital heart defects, was first approved by the Regional Committee for Medical and Health Research Ethics, Health Region West (036/09). Subsequently, the sub-study on CVDCancer has been approved (REK 
Table 2. An overview of CVDNOR hospitalizations and deaths 1994-2009.

\begin{tabular}{lrrr}
\hline & \multicolumn{1}{c}{ Men } & \multicolumn{1}{c}{ Women } & Total \\
\hline Number of patients, $n$ (\%) & $640325(48.7)$ & $674692(51.3)$ & 1315057 \\
Age at time of hospitalization, mean (sd) & $64.0(19.0)$ & $66.0(21.3)$ & $64.9(20.2)$ \\
Hospitalizations*, $\boldsymbol{n}$ (row \%) & & & \\
CVDNOR** & $2270535(53.2)$ & $1996173(46.8)$ & 4266751 \\
CVD & $1677554(54.1)$ & $1421295(45.9)$ & 3098849 \\
CHD & $750031(61.1)$ & $477909(38.9)$ & 1227940 \\
AMI & $157230(61.3)$ & $99272(38.7)$ & 256502 \\
PCI & $59781(74.7)$ & $20291(25.3)$ & 80072 \\
Cerebrovascular disease & $259102(52.8)$ & $231591(47.2)$ & 490693 \\
Diabetes mellitus & $340537(53.4)$ & $297208(46.6)$ & 637745 \\
AF & $346490(55.0)$ & $282955(45.0)$ & 629445 \\
Congenital malformations of the circulatory system & $27054(51.0)$ & $25985(49.0)$ & 53039 \\
Deaths & & & \\
Deaths among CVDNOR hospitalized patients, $n$ (\% of patients) & $234059(36.6)$ & $236836(35.1)$ & $470895(35.8)$ \\
CVD deaths among CVDNOR hospitalized patients, n (\% of patients) & $104157(16.3)$ & $111090(16.5)$ & $215247(16.4)$ \\
CVD deaths among persons not hospitalized in CVDNOR, 1994-2009 & 31192 & 37331 & 68523 \\
\hline * Hospitalizations less than 24 hours apart are merged. & & & \\
**Hospitalizations fulfilling the inclusion criteria described in Table 1. & & \\
CVD - cardiovascular disease; CHD - coronary heart disease (ICD9: 410-414, ICD10: I20-25); AMI - acute myocardial infarction (ICD9: \\
410, ICD10: I21,I22); PCI - percutaneous coronary interventions; AF - atrial fibrillation (ICD9: 427.3, ICD10: I48).
\end{tabular}

Table 3. Hospitalizations and deaths due to cardiovascular disease or diabetes mellitus among CONOR participants (19942003). A CVDNOR project 1994-2009.

\begin{tabular}{lccc}
\hline CONOR participants & Men & Women & Total \\
\hline Number of CONOR participants & 84156 & 89087 & 173243 \\
Participants who died due to CVD, $n(\%)$ & $5139(5.9)$ & $3918(4.2)$ & $9057(5.0)$ \\
Participants hospitalized with a CVD diagnosis after the CONOR survey, $n(\%)$ & $24698(29.0)$ & $19421(21.8)$ & $44118(25.5)$ \\
Participants hospitalized with a DM diagnosis after the CONOR survey, $n(\%)$ & $4313(5.1)$ & $3262(3.7)$ & $7575(4.4)$ \\
\hline
\end{tabular}

CONOR - Cohort of NORway; CVD - cardiovascular disease; DM - diabetes mellitus.

2009/861-19), as has linkages to the Hordaland Health Studies (REK 2009/825), the Norwegian Counties studies 1974-88 (REK 2012/827), and the clinical trials NORVIT-WENBIT (REK 2010/1880).

\section{Preliminary Results}

During the study period 1994-2009, 1315057 persons (51.3\% women) contributing with 4266751 hospitalizations (46.8\% among women) fulfilled the inclusion criteria listed in Table 1.

A total of 68523 patients died from CVD without being hospitalized during the same period (Table 2). Mean (SD) age for hospitalized patients was 64.9 (20.2) years. Women were older than men at the time of hospitalization. A coronary heart disease (CHD) diagnosis code was found in $1 / 3$ of the hospitalizations as main or secondary diagnosis. A DM diagnosis code was found in $15 \%$ and a cerebrovascular disease diagnosis code in $10 \%$ of all hospitalizations, and an atrial fibrillation diagnosis code was found in $14.8 \%$. During the study period, $35.8 \%$ of patients who had a CVDNOR hospitalization died. Of these, $45.7 \%$ had a
CVD related code as the underlying cause of death and $63.2 \%$ had a CVD related code mentioned on the death certificate. In CVDNOR, 53039 (4\%) patients were registered with a congenital heart defect code.

Table 3 summarizes the events (hospitalizations and deaths) for CONOR participants after participation in the health survey. Among all CONOR participants, $25.5 \%$ had at least one CVD hospitalization and 5\% died due to CVD. During follow up, $4.4 \%$ of CONOR participants had a hospitalization during which DM was coded as main or secondary diagnosis.

Additional information regarding marital status, residence, country of birth and education for patients 30 years and older included in CVDNOR at their first hospitalization is given in Table 4 . Those individuals who did not match with the Population Registry (28357 individuals; 16226 men and 12091 women) are excluded from the table.

Almost half of the patients were married and $52 \%$ lived in the South-East region, versus $10 \%$ in the North region. The majority of patients $(92.4 \%)$ were born in Norway. Only $1.3 \%$ was born in another Nordic country and the remainder $(6.3 \%)$ elsewhere. Some 
Table 4. Socio-demographic data for patients 30 years and older at first hospitalization registered in CVDNOR and with match to the Population Registry: a CVDNOR project 1994-2009.

\begin{tabular}{|c|c|c|c|}
\hline Patients with a CVDNOR diagnosis or procedure & Men & Women & Total \\
\hline Number of patients, $n(\%)$ & 570428 & 585171 & 1155599 \\
\hline \multicolumn{4}{|l|}{ Marital status*, $n(\%)$} \\
\hline Married & $369111(64.7)$ & $263507(45.0)$ & $632618(54.7)$ \\
\hline Widow(er) & $52897(9.3)$ & $191791(32.8)$ & $244688(21.2)$ \\
\hline Divorced/separated & $66502(11.7)$ & $64949(11.1)$ & $131451(11.4)$ \\
\hline Unmarried & $78987(13.9)$ & $62636(10.7)$ & $141623(12.3)$ \\
\hline Missing & $2931(0.5)$ & $2288(0.4)$ & $5219(0.5)$ \\
\hline \multicolumn{4}{|l|}{ Residence by health region $n$ (\%) } \\
\hline South-East & $312249(54.7)$ & $324409(55.4)$ & $636658(55.1)$ \\
\hline West & $110609(19.4)$ & $113482(19.4)$ & $224091(19.4)$ \\
\hline Mid & $81248(14.2)$ & $82365(14.1)$ & $163613(13.5)$ \\
\hline North & $63382(11.1)$ & $62621(10.7)$ & $137919(14.2)$ \\
\hline Missing & $2940(0.5)$ & $2294(0.4)$ & $5234(0.5)$ \\
\hline \multicolumn{4}{|l|}{ Country of birth $n(\%)$} \\
\hline Norway & $540853(94.8)$ & $553666(94.6)$ & $1094519(94.7)$ \\
\hline Nordic countries outside Norway & $7502(1.3)$ & $8083(1.4)$ & $15585(1.4)$ \\
\hline Europe outside Nordic countries & $9958(1.8)$ & $9933(1.7)$ & $19891(1.7)$ \\
\hline Outside Europe & $12053(2.1)$ & $13406(2.3)$ & $25459(2.2)$ \\
\hline Missing & $62(0.0)$ & $83(0.0)$ & $145(0.0)$ \\
\hline \multicolumn{4}{|l|}{ Education $n(\%) * *$} \\
\hline Basic or no education & $210063(36.8)$ & $271051(46.3)$ & $481114(41.6)$ \\
\hline Upper secondary education & $254005(44.5)$ & $220243(37.6)$ & $474248(41.0)$ \\
\hline Tertiary education, short & $60663(10.6)$ & $67832(11.6)$ & $128495(11.1)$ \\
\hline Tertiary education, long & $29644(5.2)$ & $10070(1.7)$ & $39714(3.4)$ \\
\hline Missing & $16053(2.8)$ & $15975(2.7)$ & $32028(2.8)$ \\
\hline
\end{tabular}

$75.7 \%$ had upper secondary education or less.

Figure 1 presents trends in hospitalizations with a CVD or DM related diagnosis or CVD procedure during the study period. Hospitalization rates per 100000 inhabitants are age-standardized using the Nordic population in the year 2000 as standard population. Both the number of hospitalizations and age adjusted rates increased from 1994 to 2006 and levelled off or even decreased thereafter.

The CVDNOR data became available for analysis around mid-2012. Our first objective was to investigate trends of acute myocardial infarction (AMI) incidence rates, by sex and age groups (Sulo et al, submitted; abstract presented at EuroPrevent, Rome, April 2013). Overall, age standardized incidence rates decreased during 2001-2009. However, among younger adults ( $<45$ years), rates of hospitalization for incident AMI increased over time. Other on-going analyses include trends in survival after AMI, trends in AMI event rates and recurrences and association between education level and survival after AMI (two abstracts accepted to ESC 2013, Amsterdam).

\section{How can CVDNOR data be accessed?}

Information on how to apply for access to CVDNOR data can be found on the study website www.cvdnor.no.

\section{Strength and weaknesses}

CVDNOR is a large dataset covering the whole population of Norway. As such it is not influenced by selection bias. However, as in other registry-based information, details on risk factors, severity of the disease, medication use and other clinical information is missing. Because patients with less severe forms of CVD or DM are not always hospitalised (or die), the estimates based on CVDNOR data will underestimate the true occurrence of these conditions. However, by linkages to the prescription registry, information on the true incidence of medically treated diseases like DM will likely be obtained.

\section{ACKNOWLEDGEMENTS}

The CVDNOR project has received economic support from Nasjonalforeningen for folkehelsen. The authors thank Tomislav Dimoski at The Norwegian Knowledge Centre for the Health Services, Oslo, Norway for his contribution by developing software necessary for obtaining data from Norwegian hospitals, conducting the data collection and quality assurance of data in this project. 

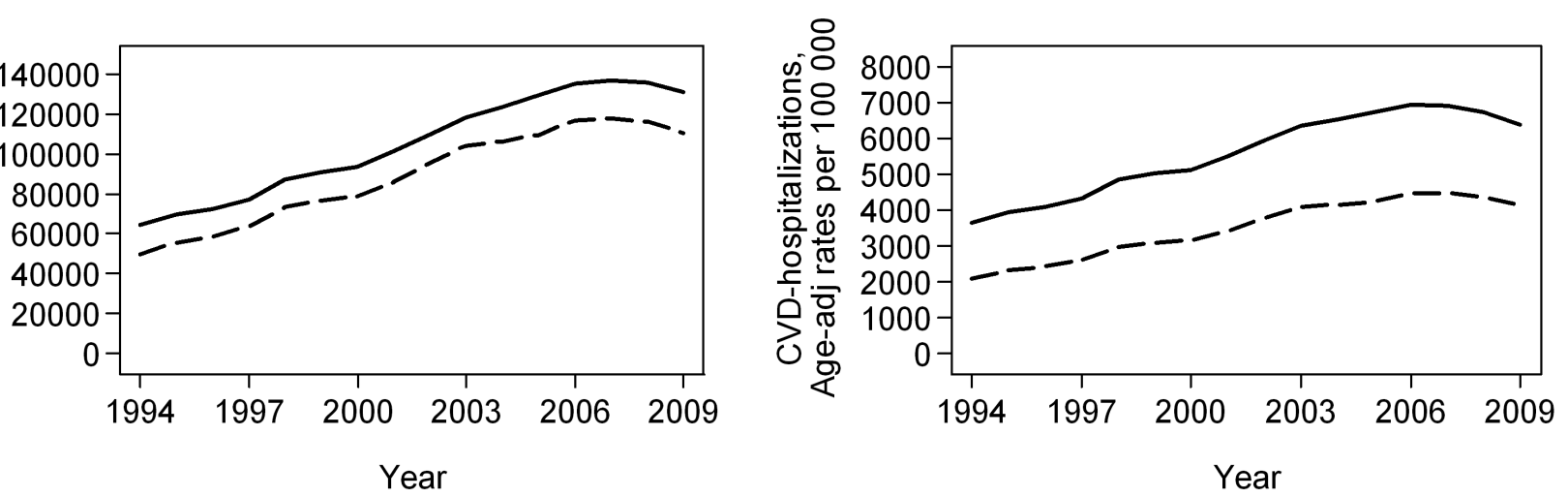

Year

\section{Men - - - Women}
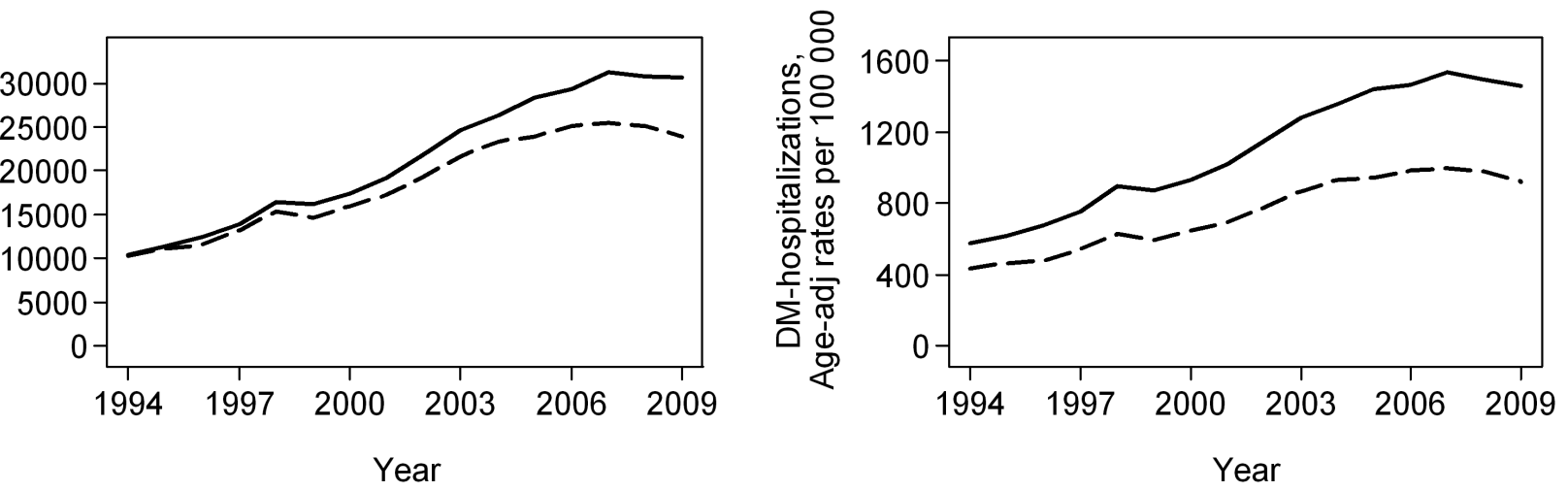

Figure 1. Trends in hospitalizations with a cardiovascular (CVD) or diabetes mellitus (DM) related diagnosis or CVD procedure, 1994-2009.

\section{REFERENCES}

1. Reikvam A, Hagen TP. Changes in myocardial infarction mortality. Tidsskr Nor Loegeforen 2011; 131 (5): 468-470.

2. Bønaa KH. Coronary heart disease mortality. Tidsskr Nor Lageforen 2011; 131 (5): 440-441.

3. Tunstall-Pedoe H. The decline in coronary heart disease; did it fall or was it pushed? BMJ 2012; $344: \mathrm{d} 7809$.

4. O'Flaherty M, Buchan I, Capewell S. Contributions of treatment and lifestyle to declining CVD mortality: why have CVD mortality rates declined so much since the 1960s? Heart 2013; 99 (3): 159-162.

5. Tunstall-Pedoe H, Kuulasmaa K, Mahonen M, Tolonen H, Ruokokoski E, Amouyel P. Contribution of trends in survival and coronary-event rates to changes in coronary heart disease mortality: 10-year results from 37 WHO MONICA project populations. Monitoring trends and determinants in cardiovascular disease. Lancet 1999; 353 (9164): 1547-1557.

6. Håheim LL, Holme I, Søgaard AJ, Lund-Larsen PG. [Changes in cardiovascular risk factors among men in Oslo during 28 years]. Tidsskr Nor Lageforen 2006; 126 (17): 2240-2245.

7. Jenum AK, Graff-Iversen S, Selmer R, Søgaard AJ. [Risk factors for cardiovascular disease and diabetes through three decades]. Tidsskr Nor Lageforen 2007; 127 (19): 2532-2536.

8. Njølstad I, Arnesen E, Lund-Larsen PG. Smoking, serum lipids, blood pressure, and sex differences in myocardial infarction. A 12-year follow-up of the Finnmark Study. Circulation 1996; 93 (3): 450-456.

9. Øyen N, Nygård O, Igland J, Tell GS, Nordrehaug JE, Irgens LM, et al. [Hospital admission rates for cardiovascular diseases in Western Norway, 1992-2001]. Tidsskr Nor Laegeforen 2008; 128 (1): 17-23.

10. Langorgen J, Igland J, Vollset SE, Averina M, Nordrehaug JE, Tell GS, et al. Short-term and long-term case fatality in 11878 patients hospitalized with a first acute myocardial infarction, 1979-2001: the Western Norway cardiovascular registry. Eur J Cardiovasc Prev Rehabil 2009; 16 (5): 621-627.

11. Mannsverk J, Wilsgaard T, Njølstad I, Hopstock LA, Løchen ML, Mathiesen EB, et al. Age and gender differences in incidence and case fatality trends for myocardial infarction: a 30-year follow-up. The Tromsø Study. Eur J Prev Cardiol 2012; 19 (5): 927-934. 
12. Håheim LL, Holme I, Hjermann I, Leren P, Tonstad S. Trends in the incidence of acute myocardial infarction and stroke: a 21 -year follow-up of the Oslo study. Scand Cardiovasc J 2004; 38 (4): 216-221.

13. Truelsen T, Mahonen M, Tolonen H, Asplund K, Bonita R, Vanuzzo D. Trends in stroke and coronary heart disease in the WHO MONICA Project. Stroke 2003; 34 (6): 1346-1352.

14. Evans A, Tolonen H, Hense HW, Ferrario M, Sans S, Kuulasmaa K. Trends in coronary risk factors in the WHO MONICA project. Int J Epidemiol 2001; 30 (Suppl 1): S35-40.

15. Omsland TK, Emaus N, Tell GS, Ahmed LA, Center JR, Nguyen ND, et al. Ten-year risk of second hip fracture. A NOREPOS study. Bone 2013; 52 (1): 493-497.

16. Clench-Aas J, Helgeland J, Dimoski T, Gulbrandsen DH, Holmboe O, Movinckel P, et al. Methodological developement and evaluation of 30-day mortality as quality indicator for Norwegian hospitals. Available at http://www.kunnskapssenteret.no/Publikasjoner/Methodological+development+and+evaluation+of +30 day+mortality+as+quality+indicator+for+Norwegian+hospitals.1246.cms. 2005: 1-198.

17. Næss Ø, Søgaard AJ, Arnesen E, Beckstrøm AC, Bjertness E, Engeland A, et al. Cohort profile: cohort of Norway (CONOR). Int J Epidemiol 2008; 37 (3): 481-485.

18. Bønaa KH, Njølstad I, Ueland PM, Schirmer H, Tverdal A, Steigen T, et al. Homocysteine lowering and cardiovascular events after acute myocardial infarction. N Engl J Med 2006; 354 (15):1578-1588.

19. Ebbing M, Bleie O, Ueland PM, Nordrehaug JE, Nilsen DW, Vollset SE, et al. Mortality and cardiovascular events in patients treated with homocysteine-lowering B vitamins after coronary angiography: a randomized controlled trial. JAMA 2008; 300 (7): 795-804.

20. Andersen TK. Klassifikasjon av medisinske prosedyrer og kirurgiske inngrep 2006. Oslo: Sosial-og helsedirektoratet, 2006. 